\title{
The diagnostic and conceptual inconsistencies of ADHD leading to false positives
}

\begin{abstract}
Attention-deficit hyperactivity disorder (ADHD) is one of the most common disorders of childhood and yet the diagnosis remains controversial. There are no specific cognitive, metabolic, neurological markers or medical tests for ADHD. Comorbidity is also extremely high, leading to doubt on the specificity of the diagnosis. Another commonly cited shortcoming of the ADHD construct is that none of the symptoms are uniquely diagnostic of ADHD and more often than not, each symptom can also be explained by other diagnoses. Specifically, children with anxiety disorders, autism spectrum, or cognitive disorders secondary to medical conditions share many common characteristics with developmental ADHD. Because the behaviorally defined DSM diagnostic system is not neuroanatomically defined, the diverse range of ADHD symptoms cannot be precisely mapped to any particular anatomic structure. In fact, imaging studies of ADHD suggests countless areas of possible dysfunction. This makes ADHD less likely to have a single etiology. Despite these multitudes of issues, ADHD has been maintained in the DSM-V with only small diagnostic modifications. Healthcare providers are encouraged to stay abreast of ongoing diagnostic research, but potentially confusing diagnosis.
\end{abstract}

Keywords: attention-deficit hyperactivity disorder, developmental disorder, misdiagnosis
Volume I Issue 5 - 2014

\author{
Robert B Perna,Ashlee R Loughan \\ TIRR Memorial Hermann, Texas Medical Center, USA
}

Correspondence: Robert B Perna, Clinical Neuropsychologist, TIRR Memorial Hermann, Texas Medical Center, 3603

Hummingbird Lane, Alvin, Texas 775II, USA, Tel 706-750-2572, Email dr.perna@juno.com

Received: August 02, 2014 | Published: September 23, 2014

\section{Abbreviations: ADHD, attention-deficit hyperactivity} disorder; DSM,diagnostic and statistical manual of mental disorders; $\mathrm{CDC}$,centers for disease control; ADD, attention-deficit disorder

\section{Introduction}

Attention-deficit hyperactivity disorder (ADHD) is one of the most common disorders of childhood ${ }^{1}$ and has become quite controversial within the clinical field of psychology, academia, and throughout society, both conceptually and neuroanatomically. The CDC has suggests that in the US, $9.5 \%$ of children ages 3 to 17 years, have ADHD. ${ }^{2}$ This prevalence of ADHD has risen above many other childhood disorders, leading to the question of possible over diagnosis and the validity of diagnostic criteria. Currently, ADHD is defined on the basis of observable or reportable behaviors within multiple environments/settings. As such, the diagnostic validity weighs solely on asking a series of questions of caregivers and teachers in the child's life regarding their daily activity. "Does the child often...?" and "Does this lead to functional impairment?" Scoring enough "yes" responses suggests the clinical diagnosis of ADHD. However, this process has become highly dependent on subjective information regarding developmentally appropriate behavior from people often not clinically trained. This process should warrant diagnostic caution given the risk of making a lifelong misdiagnosis. In discussing this challenge among colleagues, all too often you may hear a clinician state: "I undiagnose ADHD as often as I diagnose it."

\section{ADHD:Are symptoms too diverse}

There are no specific cognitive, metabolic or neurological markers or medical tests for ADHD. Comorbidity is also extremely high, undermining the specificity of the diagnosis. ${ }^{3}$ To make sense of the ADHD diagnosis, it is essential to understand its true definition. ADHD is a behaviorally defined diagnosis that is based on observable behaviors, not an etiologically or medically defined diagnosis. Unfortunately the behaviors are so diverse that multiple brain systems can be involved and differ for each individual. Essentially, the diverse range of potentially diagnostic symptoms results in patients being diagnosed with $A D H D$, yet presenting very different from one another.

ADHD is characterized by dysfunctional levels of inattentiveness, impulsivity, and hyperactivity. The Diagnostic and Statistical Manual of Mental Disorders (DSM) lists 18 possible behavioral symptoms for diagnosing the condition, ${ }^{4} 9$ observations that concern inattention, 6 symptoms concerning hyperactivity, and 3 behavioral symptoms pertaining to impulsivity. This categorical system allows for the classification of three behavioral subtypes of ADHD: Inattentive, Hyperactive-Impulsive, or Combined. Regardless of subtype, this is a highly heterogeneous population because the potential 18 symptoms can be "combined" in a variety of ways. According to reports, approximately $50 \%$ of those classified as ADHD meet the criteria for Inattentive-type, while only $20 \%$ are found to exhibit HyperactiveImpulsive Type and $30 \%$ as Combined Type.

Even more confusing is the frequently changing terms of this diagnosis. Often parents will sit across clinicians in the interview or feedback adamantly rejecting a descriptor of ADHD when their child meets the criteria for ADHD - Inattentive type. In their understanding, their child then has ADD, not ADHD. Again the dramatic difference in presentation between subtypes causes it to be assumed by some to be two different disorders. Many clinicians agree regarding the need to provide significant parent/caregiver education regarding ADHD. In fact parenting training could also significantly help symptom management.

\section{Symptoms not unique to ADHD}

Another commonly cited shortcoming of the ADHD construct is that none of the symptoms are uniquely diagnostic of ADHD and each symptom can also be explained by other diagnoses. Specifically, children with anxiety disorders, autism spectrum disorders, sleep disorders, and various medical conditions can exhibit many symptoms/ behaviors which could appear to be ADHD. Empirical research 
also suggests a variety of pre- and postnatal pathological processes which can cause symptoms similar to ADHD. Even such common condition as low birth weight has been shown to be associated with the development of ADHD symptoms. ${ }^{5}$

Symptoms in various DSM categories overlap to such a high degree that differential diagnosis becomes extremely problematic, even when clinicians are trained to eliminate variables or differentiate between specific etiologies. For example, the significant overlap of symptoms present between individuals with ADHD and / or autism. ${ }^{6}$ According to the DSM, ADHD should be determined as a differential diagnosis instead of a comorbidity of autism; as the symptoms of inattention or impulsivity are also present in those with autism. Moreover, many studies of ADHD are by necessity conducted in clinic-referred samples, where comorbidity has become the rule and not the exception increasing the challenge of researching an individual diagnosis.?

\section{ADHD and neuroanatomy}

Because the behaviorally defined DSM diagnostic system is not neuroanatomically organized, the diverse range of ADHD symptoms cannot be mapped to any particular anatomic structure. In fact, the multitude of imaging studies of ADHD suggests countless areas of possible dysfunction. This fact also makes ADHD less likely to have a single etiology, and less diagnosable by any neuropsychological test $^{8}{ }^{8}$ though frequently having the co-occurrence of executive dysfunction. ${ }^{9,10}$

One of the biggest challenges of understanding the neuropathology in ADHD is the fact that the capacity to deploy attention is supported by a network of complex functional processes in the brain. These symptoms call upon a wide range of circuits which are not only found in multiple areas of brain, but also leave the potential for many possibilities etiologies leading to dysregulation in the attention systems of the brain. ${ }^{11-15}$ In fact, recently, Bernstein ${ }^{16}$ critiqued the diagnostic construct of ADHD by pointing out the many brain systems which can account for the diverse range of diagnostic symptoms found in individuals diagnosed with ADHD and how the aforementioned ADHD behavioral triad (inattentiveness, impulsivity, $\&$ hyperactivity) can actually be the manifestation of very different underlying neuroanatomical processes. Koziol and Stevens ${ }^{8}$ added that ADHD in any given individual stems from dysfunction within one or more different neural mechanisms, possibly occurring as the result of several distinct etiological influences. So consistent with the diverse clinical presentation, there appears to be evidence and expert opinions that the neuropathology is far more complex then "checkingoff" observable symptoms.

\section{Conclusion}

ADHD was recently maintained in the DSM-V and only received small modifications to the diagnostic criteria, so it seems clear there is a consensus on the validity and utility of this diagnosis. Based on the current clinical literature the following are suggestions prior to diagnosing:

i. Do a thorough clinical interview to establish the course of diagnostic symptoms and rule out alternative explanations and factors that may be exacerbating symptoms.

ii. Review DSM diagnostic criteria with clients.

iii. Seek information from multiple informants across settings (classroom observation by a clinician is very helpful). iv. Administer standardized parent/teacher report instruments (such as the Behavioral Assessment System for Children- BASC, Child Behavior Checklist-CBCL) as a way to get age based comparisons to assess severity of symptoms.

v. Administer multiple cognitive measures focusing on attention compared to aged norms.

vi. Gather information on the parents/ guardians understanding of the diagnosis and need for appropriate education and support.

vii. Discuss and educate parents about all evidenced based treatment options.

\section{Acknowledgments}

None.

\section{Conflicts of Interest}

There is no conflict of interest.

\section{Funding}

None.

\section{References}

1. Angold A, Erkanli A, Silberg J, et al. Depression scale scores in 8-17year-olds: effects of age and gender. $J$ Child Psychol Psychiatry. 2002;43(8):1052-1063.

2. Centers for Disease Control. Attention Deficit Hyperactivity Disorder 2014.

3. Timimi S, Taylor E. ADHD is best understood as a cultural construct. $\mathrm{Br}$ J Psychiatry. 2004;184:8-9.

4. American Psychiatric Association. Diagnostic and Statistical Manual of Mental Disorders- IV (DSM-IV). 2000.

5. Pettersson E, Sjolander A, Almquist C, et al. Birth weight as an independent predictor of ADHD symptoms: a within-twin pair analysis. J Child Psychol Psychiatry. 2014.

6. Mayes SD, Calhoun SL, Mayes RD, et al. Autism and ADHD: Overlapping and discriminating symptoms. Research in Autism Spectrum Disorders. 2012;6(1):277- 285.

7. Pritchard AE, Nigro CA, Jacobson LA, et al. The role of neuropsychological assessment in the functional outcomes of children with ADHD. Neuropsychol Rev. 2012;22(1):54-68.

8. Koziol LF, Stevens MC. Neuropsychological Assessment and The Paradox of ADHD. Appl Neuropsychol Child. 2012;1(2):79-89.

9. Biederman J, Monuteaux MC, Doyle AE, et al. Impact of executive function deficits and attention-deficit/hyperactivity disorder (ADHD) on academic outcomes in children. J Consult Clin Psychol. 2004;72(5):757-766.

10. Brown TE, Reichel PC, Quinlan DM. Executive function impairments in high IQ adults with ADHD. J Atten Disord. 2009;13(2): 161-167.

11. Ashtari M, Kumra S, Bhaskar SL, et al. Attention-deficit hyperactivity disorder: A preliminary diffusion tensor imaging study. Biol Psychiatry. 2005;57(5):448-455.

12. Casey BJ, Nigg JT, Durston S. New potential leads in the biology and treatment of attention deficit-hyperactivity disorder. Curr Opin Neurol. 2007;20(2):119-124.

13. Castellanos FX, Proal E. Large-scale brain systems in ADHD: Beyond the prefrontal-striatal model. Trends Cogn Sci. 2012;16(1):17-26. 
14. Filley CM. The neuroanatomy of attention. Semin Speech Lang. 2002;23(2):89-98.

15. Shipp S. The brain circuitry of attention. Trends Cogn Sci. 2004;8(5):223230.
16. Bernstein JH. Clinical Encounters of the ADHD Kind: The Unique Role of Neuropsychology. Appl Neuropsychol Child. 2012;1(2):105-111. 